\title{
Sistema de policultivo de plantas hospederas de lepidópteros comerciales en condiciones de campo abierto en la Zona de Satipo
}

\author{
Polyculture system of host plants of lepidoptera commercial open field conditions \\ in the Area Of Satipo
}

José M. Alomía L.'

'Facultad de Ciencias Agrarias - Satipo de la Universidad Nacional del Centro del Perú.

\begin{abstract}
RESUMEN
La investigación se realizó en la zona de Satipo con el objetivo de evaluar el policultivo de plantas Pasiflora edulis, Heliconia sp. Piper angustifolia y Brumfelsia grandiflora como hospederas de mariposas. En cuanto a las características de crecimiento el maracuyá Pasiflora edulis, muestra mejores características de longitud de planta y número de hojas. El promedio de longitud de hojas para el maracuyá fue de 13,65 cm y anchura de hojas es 14,2 cm. El promedio de longitud de plantas de maracuyá fue 4,07 m y el número de hojas promedio fue de 163,5. Existe diferencia estadística significativa de longitud de hoja y anchura de hoja para la zona de Pangoa y Rio Negro. Dione juno completa su ciclo de desarrollo a los 32,8 días, el huevo incuba en 5,2 días, los estadíos larvales duran un total de 17,4 días, la prepupa 2,4 días y la pupa 7,8 días. Papilio thoas completa su ciclo de desarrollo a los 44,8 días, el huevo incuba en 4,4 días, los estadíos larvales duran un total de 19,2 días, la prepupa 2,4 días y la pupa 18,8 días. La mariposa del chirizanango completa su ciclo de desarrollo a los 30,6 días, el huevo incuba en 6,2 días, los estadíos larvales duran un total de 16,8 días, la prepupa 1,4 días y la pupa 6,2 días. El incremento larval de Dione juno hasta los 8 primeros meses, tiene un crecimiento exponencial desde 63 hasta 268 larvas en un área de 10 m2; esto implica un rendimiento de 45,1 larvas/m2 ó 451000 larvas/ha, es decir una alta rentabilidad, dado que el precio de la mariposa por unidad en el mercado tiene un promedio de U.S. \$.0, 50 haciendo un total de U.S. \$.225 500 que supera al café y a la coca. Se recomienda implementar programas de crianza de mariposas en maracuyá para el productor de la zona.
\end{abstract}

\begin{abstract}
The research was conducted in the Satipo in order to assess the plant Passiflora edulis polyculture, Heliconia sp. Piper angustifola and Brumfelsia grandiflora as host of butterflies. Regarding the growth characteristics passionfruit Passiflora edulis, best shows features of plant length and number of leaves. The average length of leaves for the passion fruit was $13,65 \mathrm{~cm}$ and leaf width is $14,2 \mathrm{~cm}$. The average length of passion fruit plants was 4,07 $\mathrm{m}$ and the average number of leaves was 163,5. There is statistically significant difference in length and width of leaf blade area for Pangoa and Rio Negro. Dione juno full development cycle to 32,8 days, the egg hatches at 5,2 days, larval stages lasting a total of 17,4 days, the prepupa and pupa 2,4 days 7,8 days. Papilio thoas complete their development cycle to 44,8 days, the egg hatches at 4,4 days, larval stages lasting a total of 19,2 days, the prepupa and pupa 2,4 days 18,8 days. Butterfly chirizanango complete their development cycle to 30,6 days, the egg hatches at 6,2 days, larval stages lasting a total of 16,8 days, the prepupa and pupa 1,4 days 6,2 days. The increase in larval Dione juno to the first 8 months, has grown exponentially since 63-268 larvae in an area of $10 \mathrm{~m} 2$, this implies a yield of 45.1 larvae/m2 or 451000 larvae / ha, is a high performance, given that the price per unit of the butterfly on the market has an average of \$ 0,50 for a total of U.S. \$ 225500 in excess coffee and coca. It is recommended to implement breeding programs for butterflies in passion fruit producer in the area.
\end{abstract}

Palabras clave | Key words: dione, brumfelsia, passiflora, piper. dione, brumfelsia, passiflora, piper. 


\section{INTRODUCCIÓN}

La alternativa más importante es hacer sostenible el campo, para ello se tiene que cambiar del monocultivo al policultivo, como es la crianza de mariposas y su exportación a través de métodos sencillos que el agricultor pueda aplicar en el campo, ya que se cuenta con recursos naturales que necesitan conocimiento científico para aplicar la ingeniería y ser explotado. En otros países de américa central se cultiva plantas hospederas de mariposas en campo abierto o cerrado; donde se cría para luego recoger los capullos y su posterior eclosión.

Tenemos plantas hospederas como el maracuyá, el matico, el platanillo y el chirizanango; los mismos que albergan especies de mariposas de buen precio en el mercado; por lo que su caza indiscriminada no es una alternativa sostenible. Ello nos ha impulsado a buscar la crianza masal bajo condiciones naturales mediante el sistema de policultivo.

Gómez (2006), manifiesta que en la actualidad, la demanda internacional de ejemplares de mariposas es básicamente generada por seis sectores: 1) Coleccionistas, 2) Artesanías e industrias de adornos, 3) Museos, 4) Compra de escamas para la fabricación de chips y pantallas de computadores, 5) Exhibición en granjas o vivarios de insectos (mariposarios), 6) Liberación de adultos en eventos (matrimonios, bautizos, grados, etc.). Los cuatro primeros sectores requieren individuos disecados y preservados, mientras que los dos últimos requieren los individuos vivos, en forma de pupas recién formadas y adultos.

En Colombia existen mariposarios con fines de producción comercial de mariposas, como lo es el de la empresa Alas de Colombia, ubicada en el Valle del Cauca, en el cual crían alrededor de 30 especies para su exportación y para venta a nivel nacional en el mercado de liberación de mariposas en eventos. Este último mercado es de gran auge a nivel doméstico en Estados Unidos y Canadá (Moreno, 1998) citado por Gómez (2006).

Al incrementarse las plantas hospederas de mariposas, se incrementan las poblaciones naturales, que de por sí presentan una relación planta-huésped muy específica que estimula a las hembras a ovipositar en éstas. Esto se mide haciendo un monitoreo del número de huevos y presencia de orugas en las plantas hospederas cultivadas y en el número de 454 adultos marPáginas 106-111 cados que son atraídos por las plantas nectaríferas. (Gómez, 2006)

Es un sistema artificial de cría intensiva que implica un alto subsidio para la sustentación productiva. Se utiliza un invernadero forrado en tela metálica fina o tul terlenka microporo blanco, de alta luminosidad. La estructura del vivario puede construirse con varillas de hierro, en madera inmunizada, PVC o aluminio y se cose la tela manualmente con hilo de nylon. El tamaño mínimo debe ser de $6 \mathrm{~m} 2$, pero el tamaño y la forma pueden variar de acuerdo al gusto y presupuesto de la persona. Puede ser cuadrado, rectangular o circular y la altura promedio puede ser de 2 a $3 \mathrm{~m}$. En su interior se siembran las plantas hospederas y algunas nectaríferas. Las plantas son cultivadas aparte en un vivero y cuando muestran talla y follaje abundante son trasladadas al vivario. (Gómez, 2006).

Tenemos nymphálidos, helicónidos, mórphidos y papiliónidos como recursos naturales de la zona de Satipo. Los papiliónidos se les conocen como mariposas cola de golondrina. Presentan usualmente prolongaciones en forma de cola en el ala posterior, ordinariamente una, pero a veces, dos o tres. Puede separárseles de otras mariposas por tener solo una vena anal en el ala posterior y por que $\mathrm{Cu}$ en el ala anterior aparece con 4 ramas de Cu. Se encuentran en esta familia Papilio crespontes Cram., P. thoas Cram., P. desmoleus $L$. como plagas de cítricos en diversos lugares del mundo. En la costa peruana se tiene a $P$. thoas Cram. y en la selva a $P$. pandion Cram. (Beingolea, 1994).

Por ello se ha planteado el siguiente problema: ¿Cuál es el comportamiento de plantas hospederas de mariposas comerciales en condiciones naturales en la zona de Satipo?. La hipótesis planteada fue la siguiente: "Existen plantas hospederas de mariposas comerciales que tienen buen comportamiento en condiciones naturales en la zona de Satipo".

Los objetivos específicos del trabajo de investigación fueron:

- Determinar las características del crecimiento de la plantas hospederas de lepidópteros en condiciones de campo.

- Determinar el ciclo de desarrollo de la mariposas en plantas hospederas de lepidópteros en condiciones de campo. 
- Determinar el rendimiento por planta durante el primer año de plantas hospederos en condiciones de campo.

\section{MATERIALES Y MÉTODOS}

El trabajo fue desarrollado en la zona de Satipo, Río Negro y Pangoa, con plantones de Pasiflora edulis, Heliconia sp. Piper angustifolia y Brumfelsia grandiflora como hospederos de la mariposa. Altitud promedio de $660 \mathrm{msnm}$, temperatura media $26{ }^{\circ} \mathrm{C}$, humedad media $85 \%$, zona de vida Bosque húmedo subtropical lluvioso.El método de investigación es tipo exploratorio, descriptivo, longitudinal mediante evaluación del crecimiento de hospederos, observación, muestreo, colección y recuperación de las mariposas. La población estuvo dada por 30 plantas de matico, 20 plantas de maracuyá, 40 plantas de platanillo, 20 plantas de chirizanango. Para la muestra se tomó 5 plantas.

El método de investigación es tipo exploratorio, descriptivo y explicativo, mediante observación, muestreo, colección y recuperación de la mariposa Papilio thoas. La población estuvo conformada por 32 plantas de matico acondicionadas en maceteros de latas de aceite de 5 galones. Para la muestra se tomó 5 hojas por planta. La variable independiente, plantas de matico, Las variables dependientes longitud de hoja, altura de planta, $\mathrm{N}^{\circ}$ de brotes, Ciclo de desarrollo, Rendimiento de larvas. Los datos fueron procesados en cuadros de porcentaje histogramas y análisis de varianza en el SPSS.

Los materiales y equipo utilizado fueron baldes, malla tul, ligas, malla "rashé", alambre galvanizado, postes de madera, clavos, fertilizante compuesto, guano de corral, alfileres, tecnopor, caja entomológica, alcohol, azúcar, agua destilada, lapicero de tinta líquida, cartulina blanca, esmalte de uñas incoloro, costal, aserrín, machete, cuaderno de campo, estereoscopio, cámara digital, cámara filmadora.

\section{RESULTADOS}

Tabla1. ANOVA sobre longitud de hojas de platanillo según zona de Pangoa y Río Negro.

\begin{tabular}{|c|c|c|c|c|c|}
\hline & $\begin{array}{l}\text { Suma de } \\
\text { cuadrados }\end{array}$ & $\mathrm{gl}$ & Media cuadrática & $\mathrm{F}$ & Sig. \\
\hline Inter-grupos & 1460,506 & 1 & 1460,506 & 14,920 &, 000 \\
\hline Intra-grupos & 4111,433 & 42 & 97,891 & & \\
\hline Total & 5571,939 & 43 & & & \\
\hline
\end{tabular}

La tabla 1, muestra alta diferencia significativa respecto a la longitud de hoja de platanillo Heliconia sp.

Tabla 2. ANOVA sobre anchura de hojas de platanillo según zona de Pangoa y Río Negro.

Anchura Hoja

\begin{tabular}{|c|c|c|c|c|c|}
\hline & $\begin{array}{l}\text { Suma de } \\
\text { cuadrados }\end{array}$ & $\mathrm{gl}$ & $\begin{array}{l}\text { Media cua- } \\
\text { drática }\end{array}$ & $F$ & Sig. \\
\hline Inter-grupos & 83,738 & 1 & 83,738 & 9,774 & ,003 \\
\hline Intra-grupos & 359,846 & 42 & 8,568 & & \\
\hline Total & 443,584 & 43 & & & \\
\hline
\end{tabular}

Fuente: SPSS 
La tabla 2, muestra alta diferencia significativa respecto a la anchura de hoja de platanillo Heliconia sp.

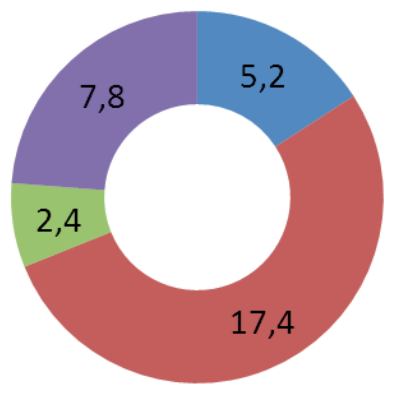

Huevo

n Larva

- Prepupa

a Pupa

Figura 1. Ciclo de desarrollo de la mariposa del maracuyá en condiciones de campo.

De la figura 1, se deduce que la mariposa del maracuyá completa su ciclo a los 32,8 días, el huevo incuba en 5,2 días, los estadíos larvales duran un total de 17,4 días, la prepupa 2,4 días y la pupa 7,8 días. Esto concuerda con lo manifestado en Costa Rica por Chacón (2001).

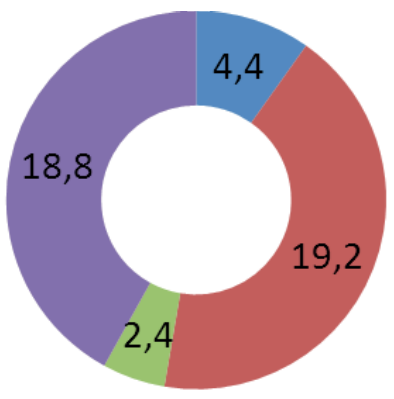

- Huevo

- Larva

- Prepur

- Pupa

Figura 2. Ciclo de desarrollo de la mariposa del matico Papilio thoas en condiciones de campo.

De la figura 2, se aprecia que la mariposa del matico completa su ciclo a los 44,8 días, el huevo incuba en 4,4 días, los estadíos larvales duran un total de 19,2 días, la prepupa 2,4 días y la pupa 18,8 días. Estos datos concuerdan con lo manifestado por Alomía (2012).

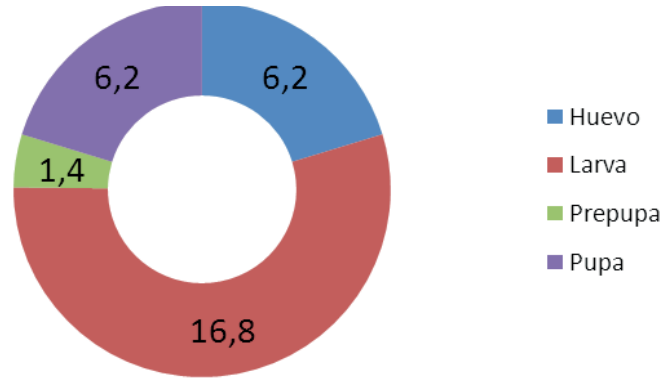

Figura 3. Ciclo de desarrollo de la mariposa del chirizanango en condiciones de campo.

De la figura 3, se aprecia que a mariposa del chirizanango completa su ciclo a los 30,6 días, el huevo incuba en 6,2 días, los estadíos larvales duran un total de 16,8 días, la prepupa 1,4 días y la pupa 6,2 días.

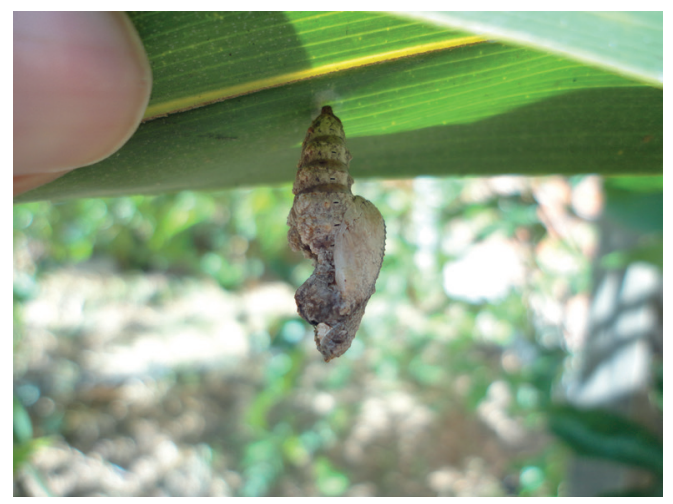

Figura 4. Pupa de la mariposa Dione juno que busca la sombra para esconder.

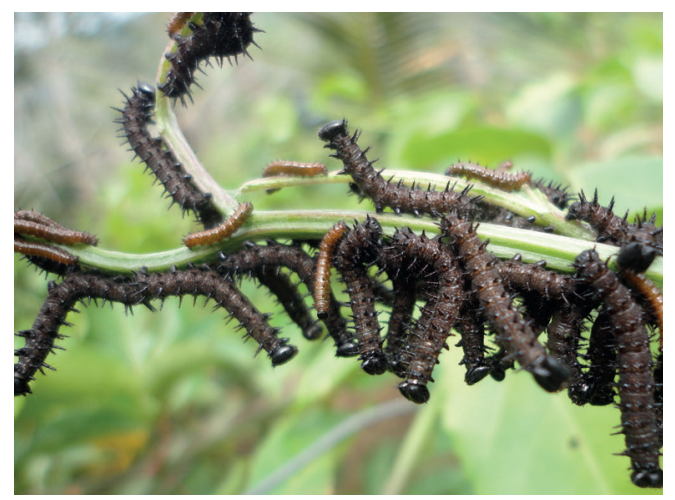

Figura 5. Larvas de Dione juno protegiendo del calor del sol. 


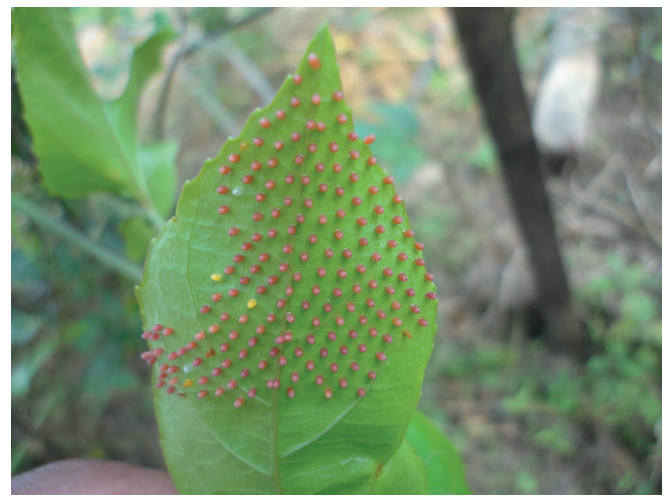

Figura 6. Posturas de Dione juno que alcanzan un promedio de 150 huevos.

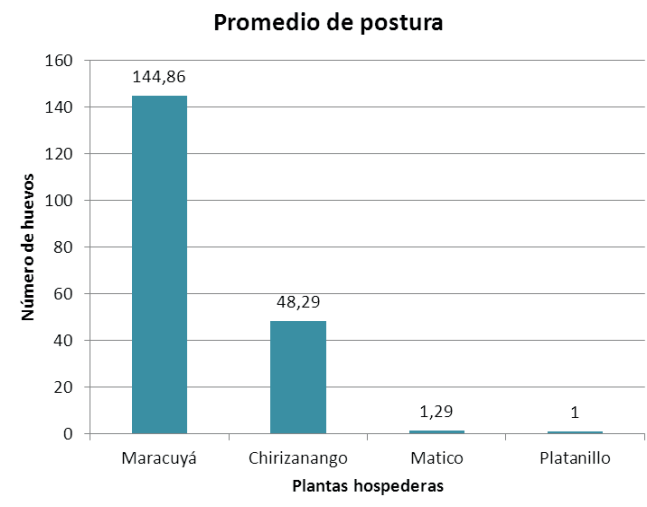

Figura 7. Promedio de posturas por hoja de las mariposas en plantas hospederas.

La figura 7, nos presenta el promedio de postura en las plantas hospederas en estudio, siendo la mariposa Dione juno del maracuyá la que presenta la mayor cantidad de huevos por colonia u hoja con 144,86 huevecillos, seguido de la mariposa del chirizanango con 48,29 huevecillos por colonia u hoja. En el matico la mariposa pone entre uno a dos huevecillos por hoja; de igual manera en el platanillo que pone un solo huevo por hoja.

El incremento larval de Dione juno sobre plantas de maracuyá hasta los 8 primeros meses, la misma que tiene un crecimiento exponencial desde 63 hasta 268 larvas en un área de 10 m2, es decir 451 larvas por esa unidad de área; esto implica un rendimiento de 45, 1 larvas/m2 en los tres primeros meses de producción.

\section{DISCUSIÓN}

El promedio de longitud de hojas para el maracuyá fue de $13,65 \mathrm{~cm}$ y anchura de hojas es $14,2 \mathrm{~cm}$, lo que muestra una hoja de forma casi redonda en los diámetros, pero con tres lóbulos bien definidos. El promedio de longitud de plantas de maracuyá fue 4,07 m y el número de hojas promedio fue de 163,5. Esto muestra un buen desarrollo de la planta dado a las condiciones de clima y suelo que se tiene en la zona, la misma que fue favorecida por el tutorado con alambre y postes que se tuvo que instalar.

El promedio de longitud de hojas de platanillo fue $39,80 \mathrm{~cm}$ y anchura de hojas fue $11,44 \mathrm{~cm}$, lo que muestra una hoja de forma alargada. El promedio de altura de plantas de platanillo fue $2,29 \mathrm{~m}$ y el número de hojas promedio fue de 8,13 . Esto muestra un buen desarrollo de la planta dado a las condiciones de clima y suelo que se tiene en la zona, y el platanillo es una planta silvestre en la zona en sus distintas variedades.

El promedio de longitud de hojas de matico fue de 14,28 cm y anchura de hojas fue $5,23 \mathrm{~cm}$ lo que muestra una hoja de forma alargada. El promedio de altura de plantas de matico fue $43,25 \mathrm{~cm}$ y el número de hojas promedio fue de $41,5 \mathrm{~cm}$. Esto muestra un buen desarrollo de la planta dado a las condiciones de clima y suelo que se tiene en la zona, y el matico es una planta silvestre en la zona en sus distintas variedades. Esto concuerda con los datos obtenidos por Alomía (2012).

El promedio de longitud de hojas de chirizanango Brumfelsia grandiflora es $11,80 \mathrm{~cm}$ y anchura de hojas es $3,78 \mathrm{~cm}$, lo que muestra una hoja de forma alargada. El promedio de altura de plantas de chirizanango fue $31,5 \mathrm{~cm}$ y el número de hojas promedio fue de 59,5. Esto muestra un buen desarrollo de la planta dado a las condiciones de clima y suelo que se tiene en la zona, y el chirizanango es una planta silvestre en la zona.

Existe alta diferencia estadística significativa para longitud de hojas y anchura de hojas respecto al platanillo Heliconius sp. en la zona de Pangoa y Río Negro, dado que Pangoa tuvo 
mejores condiciones de sombra y suelo. La asociatividad de cultivos y la materia orgánica favorecen el desarrollo de las plantas.

La mariposa del maracuyá Dione juno completa su ciclo a los 32,8 días, el huevo incuba en 5,2 días, los estadíos larvales duran un total de 17,4 días, la prepupa 2,4 días y la pupa 7,8 días.

La mariposa del platanillo Morpho sp. se ha encontrado posturas individuales, larvas solitarias desde el primer hasta el último estadío, se hizo empupar, pero no se logró emerger la mariposa. Es una mariposa muy delicada para criar, por ello no se logró calcular el ciclo de desarrollo.

La mariposa del matico Papilio thoas completa su ciclo a los 44,8 días, el huevo incuba en 4,4 días, los estadíos larvales duran un total de 19,2 días, la prepupa 2,4 días y la pupa 18,8 días. Esto concuerda con los datos obtenidos por Alomía (2012).

La mariposa del chirizanango completa su ciclo a los 30,6 días, el huevo incuba en 6,2 días, los estadíos larvales duran un total de 16,8 días, la prepupa 1,4 días y la pupa 6,2 días.

El incremento larval de Dione juno sobre plantas de maracuyá hasta los 8 primeros meses, tiene un crecimiento exponencial desde 63 hasta 268 larvas en un área de $10 \mathrm{~m} 2$. Haciendo un rendimiento total de es decir 451 larvas por esa unidad de área; esto implica un rendimiento de 45,1 larvas $/ \mathrm{m} 2$. Si se expresa en hectárea sería 451000 larvas como rendimiento potencial, que al ser cosechados para su empupamiento y obtención de mariposas tendríamos una alta rentabilidad, dado que el precio de la mariposa por unidad en el mercado tiene un promedio de U.S. \$.0,50 haciendo un tota de U.S. \$.225 500 que supera al café y a la coca. La mariposa como plaga seria de este cultivo muestra buenas características para su explotación. La planta también muestra buena capacidad de compensación.

En cuanto al promedio de postura en las plantas hospederas en estudio la mariposa Dione juno del maracuyá es la que presenta la mayor cantidad de huevos por colonia $u$ hoja con 144,86 huevecillos, seguido de la mariposa del chirizanango con 48,29 huevecillos por colonia $u$ hoja. En el matico la mariposa pone entre uno a dos huevecillos por hoja; de igual manera en el platanillo que pone un solo huevo por hoja. Esto significa que el maracuyá y el matico son

Páginas 106-111 las plantas que muestran la mayor cantidad de larvas.

\section{AGRADECIMIENTO}

A los estudiantes de la EAP Agronomía Tropical: William Aucatoma Huarancca y Rudy Pérez Sollier del VIII semestre 2013 de

Agronomía Tropical, a los estudiantes del VI Semestre 2013 de Agronomía Tropical, al IV semestre 2012 de la Sede Pangoa representado por su delegado Apolinario Zanabria, por su participación en los trabajos de campo.

\section{REFERENCIAS}

Alomía, L. José M. (2012). "Crianza masal de mariposas Papilio thoas en plantas hospederas de matico Piper angustifolia en condiciones naturales de la zona de Satipo". Facultad de Ciencias Agrarias de la Universidad Nacional del Centro del Perú.

Beingolea G. O. (1992). Guía para la identificación de insectos de interés agrícola. RAAA, Red de Acción al Uso de Agroquímicos, Lima, Perú.

Gómez, S. R. (2006). Plan de manejo propuesto para la cría de mariposas promisorias como alternativa productiva para comunidades indígenas de la Amazonia colombiana. Boletín Sociedad Entomológica Aragonesa, n 138 (2006): 451-460. Entomología Aplicada. Bogotá, Colombia. 